\title{
POSITION CONTROL OF A LEVITATING MAGNETIC ACTUATOR APPLICATIONS TO MICROSYSTEMS
}

\author{
Jiri Stepanek ${ }^{1,2}$, Hervé Rostaing ${ }^{2}$, \\ Suzanne Lesecq ${ }^{3}$, \\ Jérôme Delamare ${ }^{2}$, Orphée Cugat ${ }^{2}$ \\ ${ }^{1}$ Corresponding author: \\ Jiri.Stepanek@leg.ensieg.inpg.fr \\ ${ }^{2}$ Laboratoire d'Electrotechnique de Grenoble, France \\ BP 46, 38402 Saint Martin d'Hères Cedex \\ Tel: $+33(0) 476827154$ Fax : +33(0)4 76826300 \\ ${ }^{3}$ Laboratoire d'Automatique de Grenoble, France \\ BP 46, F-38402 Saint Martin d'Hères Cedex \\ Tel: +33 (0)4 76826225 Fax : +33 (0)4 76826388
}

\begin{abstract}
This paper deals with a novel magnetic microactuator. Its moving part is a permanent magnet in unstable levitation between two stable positions, and thus its central position must be precisely controlled. A cm-scale prototype was built to test the dynamic behaviour of the device. The quaternion algebra is used to describe the 3D movement of the moving magnet. A PD controller for position control is determined by a pole placement; it is then tested on the complex model and the theoretical results are satisfactory. Copyright $($ C 2002 IFAC
\end{abstract}

Keywords: Actuators, Electromagnetic device, Mechanical manipulator, Microsystem, Multi-degree-of-freedom system, Moment of inertia, PD Controller, Position control, Positioning system, Quaternion.

\section{INTRODUCTION}

With the growing world of MEMS, new challenges are appearing in the field of robotics. The potential applications of our device are for instance the precise micro-manipulation in biotechnology or in other fields (Cugat et al., 2003; Rostaing et al., 2004). In this case the requirements are $\mu \mathrm{m}$-scale precision and a "high" reaction speed. The control of such a microactuator raises problems of position sensing, robustness against pertubations, and controller speed.

This article presents such a magnetic micro-actuator. The application (device and position sensor) is first presented, the simplified and complete description of the system is given; the controller synthesis and some simulated results are shown in the end.

\section{APPLICATION}

The structure shown in Fig. 1, 2 and 3 is used for micro-positioning, and is protected by an international patent (Delamare et al., 2001). The actuating part is a free-moving magnet (2), which is captive of the magnetic field of two neighboring fixed magnets (1).

Here, all three magnets are magnetized in the same direction. The mobile magnet has two stable positions, against either one of the fixed magnets. Commutation between the stable positions is forced by current pulses through the conductors (3). 


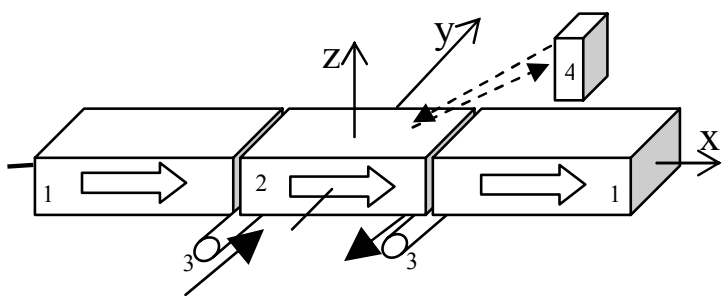

Fig. 1. Magnetic micro-actuator

The position of the mobile magnet should be controlled anywhere between these two stable positions, including the unstable equilibrium point in the middle of the device, where any small perturbation will provoke its attraction back to one of the stable positions. We control this position by modulating the current in the conductors, using feedback from the position sensor (4).

In order to achieve real-time position control, it is first necessary to determine its dynamic behaviour by calculating the $3 \mathrm{D}$ forces and torques which affect the mobile magnet in any position and/or orientation (Stepanek et al, 2004).

\subsection{Microrelay realisation}

A $\mu \mathrm{m}$-scale prototype is actually fabricated in the Laboratoire d'Electronique de Technologie de l'Information (LETI) in Grenoble, France. The actuator operates in bistable mode as a micro-relay (Fig. 2).

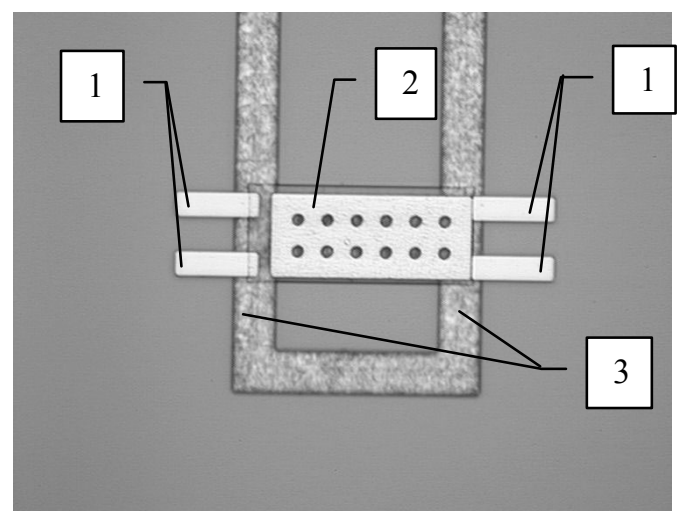

Fig. 2. Realisation of $\mu$-relay - LETI

The dimensions of the device are $50 \times 200 \mu \mathrm{m}$.

\subsection{Macroscopic prototype}

A cm-scale prototype has been built to validate the functionality and validate the model of the complete system. The prototype has two major parts: the actuator itself (Fig. 3) and a position sensor.

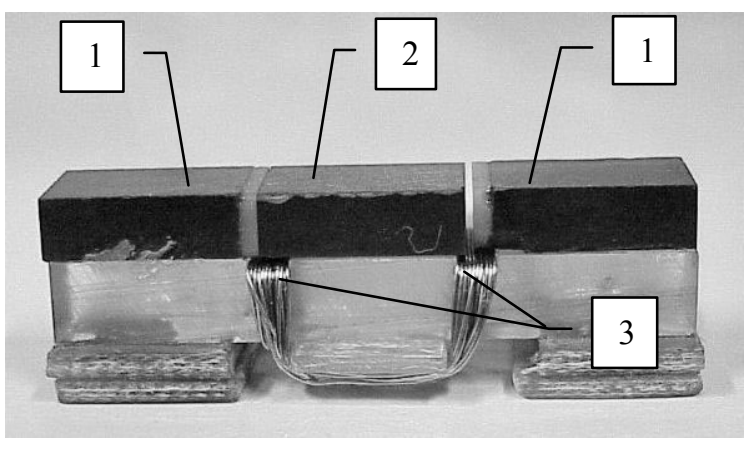

Fig. 3. Macroscopic prototype of the micro-actuator

The overall length of the prototype is $4 \mathrm{~cm}$. The conductor is made by 30 loops of copper wire. The magnets are standard ferrites, and mobile magnet in the middle can move in $0.5 \mathrm{~mm}$. Here the gap is widened with plastic stoppers so as to adjust for the strength of the magnets at this large scale.

\subsection{Position sensor}

The position of the mobile magnet is captured with a differential capacitive position sensor (Fig. 4). Each half-sensor is composed of a pair of fixed electrodes (1) facing a mobile electrode (2).

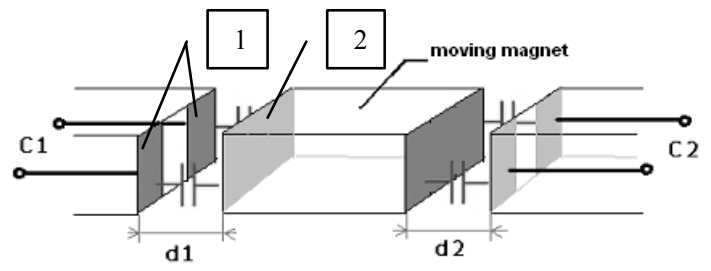

Fig. 4. Principle of the position sensor

The electronic circuit shown on Fig. 5 produces a signal proportional to the position of the moving magnet.

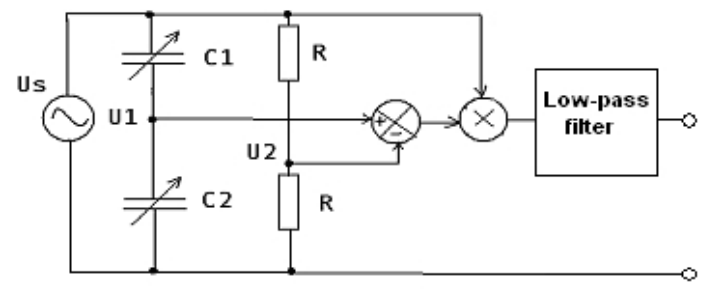

Fig. 5. Electrical diagram of the position sensor

The overall set-up is shown on Fig. 6. One of the fixed magnets (1) and the moving magnet (2) stuck on the adjustable console (5) can be seen. This configuration allows us to characterise the sensor's performance. The position signal is captured with the split electrodes (4a) and processed by the electronics (4b). The present sensor has a good resolution (100 $\mathrm{mV} / 100 \mu \mathrm{m})$, but still exhibits some linearity problems. Work is in progress on its improvement. 


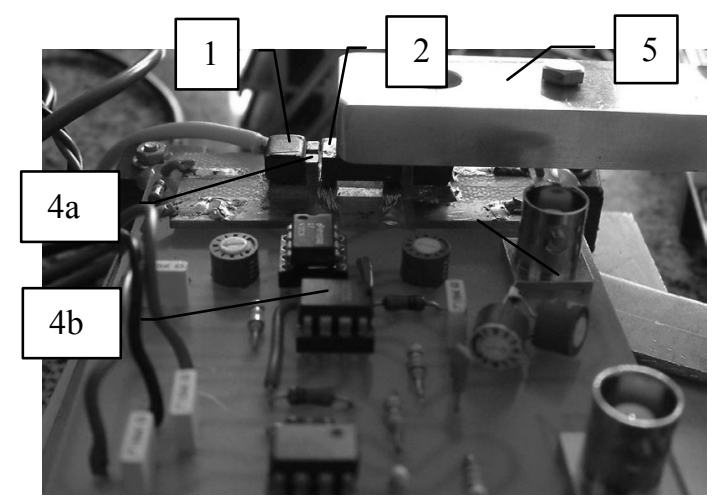

Fig. 6. Actuator \& sensor set-up

\section{SIMPLIFIED MECHANICAL SYSTEM DESCRIPTION}

The micro-actuator will be levitating in the magnetic field. It will possess six degrees of freedom. As there is a risk of possible mechanic oscillations, the complete translation / rotation movements must be modelled.

As the complete model is non-linear and too complex and cannot be fully described analytically (only linearised), a simplified description of the actuator is used to synthetise the controller and make the first tests. The developed corrector will be then tested on the complex model described in section 4 , to verify all the performance.

Let us first study the system without rotations and moving only along the $x$ direction.

The overall force exerted on the moving magnet is composed of the forces from the fixed magnets and from the coil. Dieppedale et al. (2004) showed that in the middle position, the composed force from the symmetrical fixed magnet is proportional to the moving magnet displacement. The force from the coil is proportional to the current in the coil:

$$
F_{x}=k_{1} x+k_{2} I
$$

with:

$x .$. the moving magnet's position

I.. the current in the coil

The constants $\mathrm{k}_{1}$ and $\mathrm{k}_{2}$ are the electromagnetic constants which can be easily estimated with electromagnetic calculus.

The differential equation describing the dynamics is:

$$
m \ddot{x}=k_{1} x+k_{2} I
$$

where $m$ is the moving magnet's mass.

The resulting transfer function is given by:

$$
T . F_{\text {M.S. }}=\frac{x}{I}=\frac{\frac{k_{2}}{k_{1}}}{\frac{m}{k_{1}} p^{2}-1}=\frac{k_{2}}{k_{1}} \cdot \frac{1}{\left(\tau^{2} p^{2}-1\right)}
$$

(3) corresponds to a second order unstable system. The phase is $180^{\circ}$ for all frequencies.

\section{COMPLEX ACTUATOR MODELING}

Among different approaches, the Euler angles or the quaternion formulation can be chosen in order to model a free three dimensions (3D) rotation. However, in this work, the quaternion approach has been chosen to avoid the Gimbal lock effect (Guiziou, 2002; Chou, 1992).

Let us define a fixed coordinate system (Xs, Ys, Zs), called Ro and a moving coordinate system (Xi, Yi, $\mathrm{Zi}$ ) as specified in Fig. 7.

The levitating magnet is made of a homogeneous material in the form of a rectangular parallelepiped. Its dimensions are length $a$, width $b$ and height $c$.

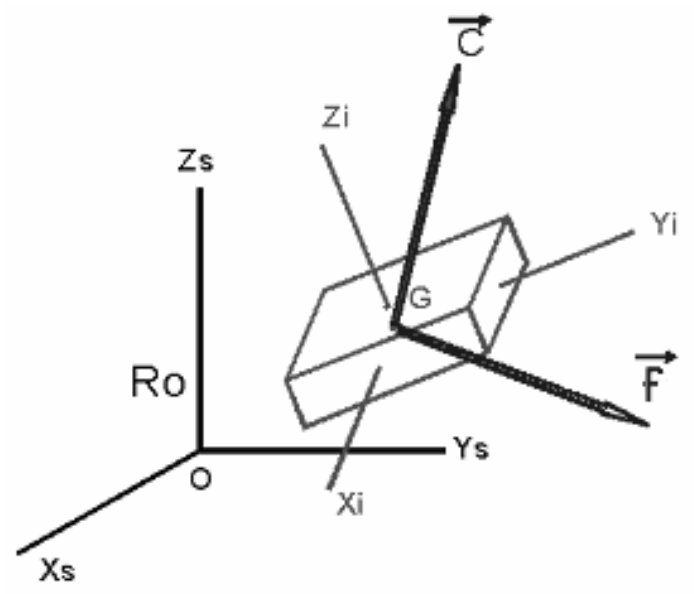

Fig. 7. Coordinate systems

The inertial moment tensor in the moving coordinates can be calculated as:

$$
I i=\left(\begin{array}{ccc}
I_{1} & 0 & 0 \\
0 & I_{2} & 0 \\
0 & 0 & I_{3}
\end{array}\right)=\frac{1}{m}\left(\begin{array}{ccc}
b^{2}+c^{2} & 0 & 0 \\
0 & c^{2}+a^{2} & 0 \\
0 & 0 & a^{2}+b^{2}
\end{array}\right)
$$

with $m$ the mass of the moving magnet.

\subsection{Dynamics: introduction}

The dynamic system contains four unknown vectors, namely:

- the translation position vector $\vec{R}$ (3 components)

- the translation speed vector $\vec{V}$ (3 components)

- the quaternion $\vec{Q}$ (4 components)

- the angular speed $\vec{\Omega}$ (3 components) 
Their components are respectively:

$$
\vec{R}=\left(\begin{array}{c}
x \\
y \\
z
\end{array}\right) \quad \vec{V}=\left(\begin{array}{c}
v x \\
v y \\
v z
\end{array}\right) \quad \vec{Q}=\left(\begin{array}{c}
q 0 \\
q 1 \\
q 2 \\
q 3
\end{array}\right) \quad \vec{\Omega}=\left(\begin{array}{l}
p \\
q \\
r
\end{array}\right)
$$

\subsection{Force and torque calculation}

The calculation is based on the magnetostatic calculus of forces exerted on a magnet in a magnetic field. If the magnet exact position, rotation and the current in the coil are known, the magnetic force and torque can be found. The magnetic force is formulated in the fixed coordinate system, the torque must be recalculated in the moving coordinate system.

Note that the calculus based upon the "simple" magnetostatic hypothesis; the magneto-dynamics effects on the systems are not considered because the Eddy currents can be neglected.

The calculus of the force $\vec{F}$ and the torque $\vec{\Gamma}$ can be seen as a "black box", function of the magnet position and rotation. They are not described in this paper and the reader can consult Stepanek et al. (2003); (2004).

$\vec{f}$ and $\vec{C}$ are the normalised force and torque:

$$
\begin{gathered}
\vec{F}=\vec{F}(R, Q, I)=\left(\begin{array}{l}
F_{1} \\
F_{2} \\
F_{3}
\end{array}\right) \quad \vec{f}=\frac{\vec{F}}{m}=\left(\begin{array}{l}
\frac{F_{1}}{m} \\
\frac{F_{2}}{m} \\
\frac{F_{3}}{m}
\end{array}\right) \\
\vec{\Gamma}=\vec{\Gamma}(R, Q, I)=\left(\begin{array}{l}
\Gamma_{1} \\
\Gamma_{2} \\
\Gamma_{3}
\end{array}\right) \quad \vec{C}=\left(\begin{array}{l}
C_{1} \\
C_{2} \\
C_{3}
\end{array}\right)=\left(\begin{array}{l}
\frac{\Gamma_{1}}{I_{1}} \\
\frac{\Gamma_{2}}{I_{2}} \\
\frac{\Gamma_{3}}{I}
\end{array}\right)
\end{gathered}
$$

\subsection{Translation equations}

The translation equations (8) and (9) are easy to understand. They obey Newton's law, using the initial conditions (10), (11):

$$
\begin{aligned}
& \frac{d \vec{R}}{d t}=\vec{v} \quad \text { (8) } \quad \frac{d \vec{v}}{d t}=\vec{f} \\
& \vec{R}_{0}=\left(\begin{array}{c}
x_{0} \\
y_{0} \\
z_{0}
\end{array}\right) \quad(10) \quad \vec{v}_{0}=\left(\begin{array}{c}
v x_{0} \\
v y_{0} \\
v z_{0}
\end{array}\right)
\end{aligned}
$$

4.4. Rotation equations - movement around the centre of gravity

The problem to be solved is given by:

$$
\vec{\Gamma}=I_{i} \cdot \frac{d \vec{\Omega}}{d t}+\vec{\Omega} \times I i \cdot \Omega
$$

The equation (12) can be rewritten:

$$
\frac{d \vec{\Omega}}{d t}=C-\left(\begin{array}{l}
\frac{I_{3}-I_{2}}{I_{1}} q r \\
\frac{I_{1}-I_{3}}{I_{2}} p r \\
\frac{I_{2}-I_{1}}{I_{3}} p q
\end{array}\right)
$$

where the initial conditions are (14) :

$$
\vec{\Omega}_{0}=\left(\begin{array}{c}
p_{0} \\
q_{0} \\
r_{0}
\end{array}\right)
$$

\subsection{Quaternions}

The quaternion $\vec{Q}$ represents the geometric rotation transforming the basis $\mathrm{R}_{0}$ into the basis $\mathrm{R}$. The quaternion multiplication is used in order to determine the elements of one vector in both coordinate systems.

The quaternion evolution has to be calculated in parallel with the instantaneous angular speed (Giuziou, 2002). These computations with initial conditions give:

$$
\begin{gathered}
\frac{d \vec{Q}}{d t}=\frac{1}{2} \cdot\left(\begin{array}{cccc}
0 & -p & -q & -r \\
p & 0 & r & -q \\
q & -r & 0 & p \\
r & q & -p & 0
\end{array}\right) \\
\vec{Q}_{0}=\left(\begin{array}{c}
q 0_{0} \\
q 1_{0} \\
q 2_{0} \\
q 3_{0}
\end{array}\right) \quad(16)
\end{gathered}
$$

Note that the quaternion must be unitary in order to represent a rotation. Therefore, it must satisfy:

$$
\sqrt{q 0^{2}+q 1^{2}+q 2^{2}+q 3^{2}}=1
$$

\subsection{Complete system}

The applied torque depends on the translation and the rotation of the moving magnet in the fixed field. No equation can be uncoupled, and the problem will 
contain 13 differential equations (Eqs. (8), (9), (13),

(15)) with 13 unknowns namely:

$$
(x, y, z, v x, v y, v z, p, q, r, q 0, q 1, q 2, q 3)
$$

One algebraic constraint (17) must also be dealt with. The complete system is numerically solved with a Matlab-Simulink block-diagram. A $4^{\text {th }}$ order fixedstep Runge-Kutta integration scheme has been chosen. Note that a variable-step method based on an explicit Runge-Kutta $(4,5)$ formula (ODE45 in Matlab) has also been tested. The fixed step has been carefully chosen thanks to the results obtained with the variable-step method.

\section{CLOSED LOOP SYSTEM}

Different works on bistable levitating systems control still exist. Faure et al. (1999) showed a corrector synthesis for a magnetic bearing where the controller zeros are chosen thanks to the system poles.

In this paper, the controller synthesis is made by the pole-placement technique (DeLarminat, 2002). The denominator of the closed-loop transfer function in then simplified to a $3^{\text {rd }}$ order non-oscillating system.

The closed loop from the Fig. 8 will be used for the synthesis.

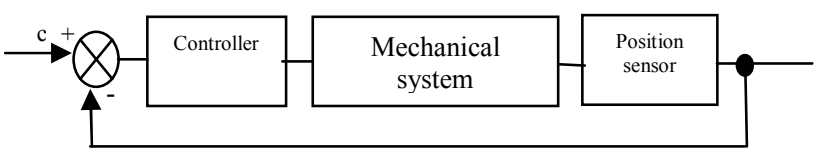

Fig. 8. Generalised closed loop system

\subsection{Controller choice and determination}

The role of the controller is firstly to regulate the moving magnet of the system in the middle position. It will also compensate the perturbations. On a further level, the controller should also be able to stabilise the moving magnet in any required position on the $\mathrm{x}$ axis (Fig.1). The other axes are not controlled in this configuration.

For the present application, a (filtered) PD controller can satisfy these constraints. It can reduce the $180^{\circ}$ phase of the system. Its transfer function is given by:

$$
T . F_{R E G}=\frac{k_{0} \cdot\left(1+\tau_{1} p\right)}{\left(1+\tau_{2} p\right)} \cdot \frac{k_{c}}{1+\tau_{c} p}
$$

Note that this transfer function is composed by the (filtered) PD controller and a first-order system which represents the limitation of the controller to supply the electric current at high frequencies. Actually, $\tau_{\mathrm{c}}<<\tau_{2}$.

\subsection{Position sensor}

The $\mathrm{x}$ position sensor has been described in section 2.3. Its transfer function is given by:

$$
T \cdot F_{P S}=\frac{k_{f}}{\left(1+\tau_{f} p\right)^{2}}
$$

Note that its characteristics satisfy $\tau_{\mathrm{f}}<<\tau_{2}$.

\section{PD CONTROLLER SYNTHESIS}

\subsection{Controller for the simplified mechanical model}

The description of the simplified mechanical system is used for the controller synthesis.

The closed-loop system transfer function is calculated with the controller transfer function (18), the mechanical system transfer function (3) and the position sensor transfer function (19). The denominator of the closed loop transfer function is a $6^{\text {th }}$ order polynom:

$$
\begin{aligned}
& \operatorname{Den}_{C . L .}(p)=K_{D e n} \cdot\left\{a_{0}+a_{1} p+\right. \\
& \left.a_{2} p^{2}+a_{3} p^{3}+a_{4} p^{4}+a_{5} p^{5}+a_{6} p^{6}\right\}
\end{aligned}
$$

where:

$$
\begin{aligned}
& K_{\text {Den }}=\frac{1}{k_{0} k_{c} k_{2} k_{f}-k_{1}} \\
& a_{0}=k_{0} k_{c} k_{2} k_{f}-k_{1} \\
& a_{1}=-2 k_{1} \tau_{f}-\tau_{c} k_{1}-\tau_{2} k_{1}+k_{0} k_{c} k_{2} k_{f} \tau_{1} \\
& a_{2}=\tau^{2} k_{1}-k_{1} \tau_{f}^{2}-2 \tau_{c} k_{1} \tau_{f}-\tau_{2} \tau_{c} k_{1} \\
& a_{3}=2 \tau^{2} k_{1} \tau_{f}+\tau_{c} \tau^{2} k_{1}-\tau_{c} k_{1} \tau_{f}^{2}+\tau_{2} \tau^{2} k_{1}-\tau_{2} k_{1} \tau_{f}^{2}-2 \tau_{2} \tau_{c} k_{1} \tau_{f} \\
& a_{4}=\tau^{2} k_{1} \tau_{f}^{2}+2 \tau_{c} \tau^{2} k_{1} \tau_{f}+2 \tau_{2} \tau^{2} k_{1} \tau_{f}+\tau_{2} \tau_{c} \tau^{2} k_{1}-\tau_{2} \tau_{c} k_{1} \tau_{f}^{2} \\
& a_{5}=\tau_{c} \tau^{2} k_{1} \tau_{f}^{2}+\tau_{2} \tau^{2} k_{1} \tau_{f}^{2}+2 k_{1} \tau^{2} \tau_{2} \tau_{f} \tau_{c} \\
& a_{6}=\tau_{2} \tau_{c} \tau^{2} k_{1} \tau_{f}^{2}
\end{aligned}
$$

As stated previously, Den $n_{C . L \text {. }}$ is simplified to as $3^{\text {rd }}$ order polynom with:

$$
\operatorname{Den}_{P . P .}=(\alpha p+1)^{3}
$$

Because of the application context, an overshoot on the position of the magnet is not suitable, the response time being of second order interest. This main requirement can justify the choice of (22).

Using and optimisation solver, (for instance the

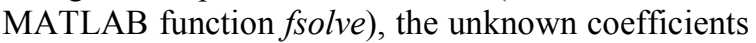
defining the controller $k_{0}, \tau_{1}$ and $\tau_{2}$ are computed for any chosen $\alpha$. Actually, the validity of the simplification hypothesis (21) depends on the numerical value set for $\alpha$. Therefore, after the optimisation step, the validity of the hypothesis (22) must be a posteriori checked. 
6.2. Application of the obtained controller onto the complex actuator model

The controller obtained is now tested on the complete quaternion model to validate the behaviour of the whole actuator. Some results are shown on Fig. 9 :
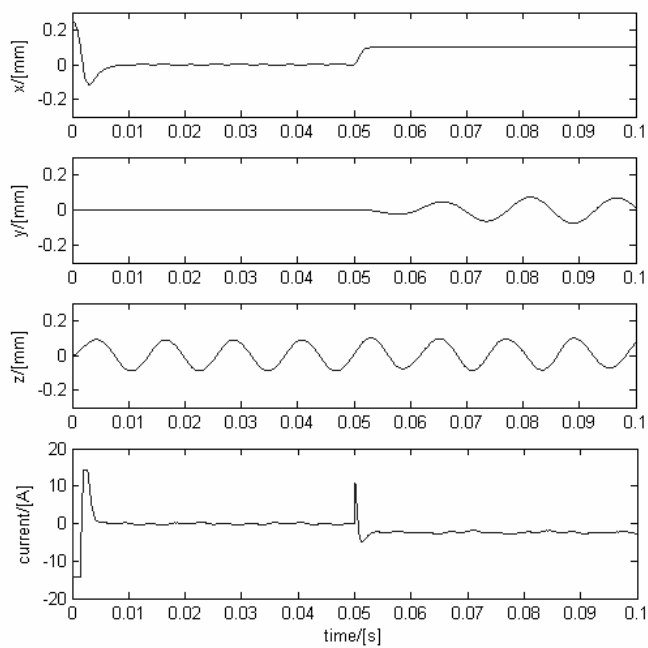

Fig. 9. Simulation results : $\mathrm{X}, \mathrm{Y}$ and $\mathrm{Z}$ translations and control current versus time

The position on the $\mathrm{x}$-axis is successfully controlled from $0 \mathrm{~mm}$; at $\mathrm{t}=50 \mathrm{~ms}$ a step of $0.1 \mathrm{~mm}$ has been applied. The actuator oscillates in the z-axis direction with an amplitude of $0.1 \mathrm{~mm}$. The oscillation is generated by coupled forces created by conductor 3 , (Fig. 1.) and are not attenuated because there is no friction in the model. However, in practice it may be dampened by air. A similar phenomenon can be observed in the y-axis since 50ms.

An x-position overshoot can be seen on Fig. 9. It is due to two non-linear events: current saturation and position saturation. There is no overshoot in the step response at $50 \mathrm{~ms}$.

The rotation movement is represented in Fig. 10. Quaternions make it difficult to imagine the actuator behaviour from the graphs - but it is clearly visible on animations. The actuator exercises a complicated "Lissajoux-type" periodic rotation around its centre of gravity.

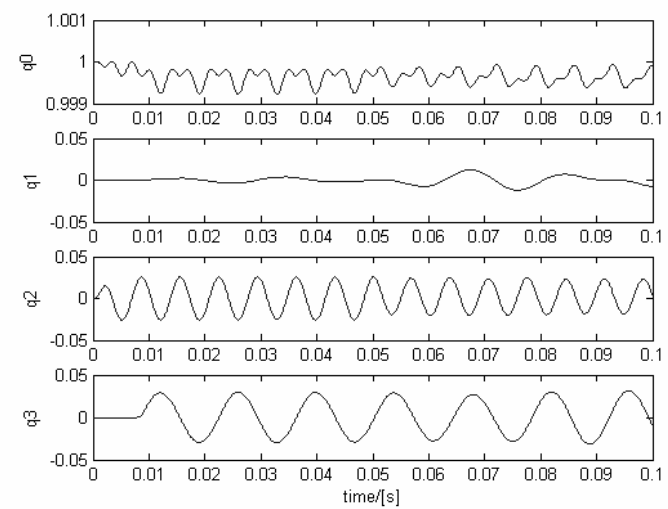

Fig. 10. Simulation results - quaternions vs time

\section{CONCLUSION}

The complete description of the kinematics and dynamics of the novel levitating magnetic microactuator has been shown. The modelling is made on a $\mathrm{cm}$-scale prototype, but can be easily applied on the micrometric integrated device. The complex actuator movement can be simulated with this tool.

A PD controller was then established to control the actuator movement. A simplified model was used for the synthesis. The resulting controller was tested on the quaternion model and the results are promising.

The controller has been realised in electronics and tested on the magnetic system. The mobile magnet is successfully maintained in levitation.

\section{REFERENCES}

Chou J.C.K, (1992). Quaternion Kinematic and Dynamic Differential Equations. Vol. no. 8, in: IEEE Transactions on robotics and automation.

Cugat O., J. Delamare, G. Reyne, (2003). MAGnetic Micro-Actuators \& Systems MAGMAS. Vol. no. 39-6, pp 3608-3612, in: IEEE Transactions on Magnetics,

Delamare, J., O. Cugat, C. Locatelli, (2001). Microactionneur bistable utilisant le guidage magnétique et des aimants. $\mathrm{N}^{\circ}$ FR $01 \quad 10$ 81, International patent

DeLarminat, P. (2002). Commande des systèmes linéaires. Hermès, Lavoisier, ISBN 2-74620492-4

Dieppedale C., B. Desloges, H. Rostaing, J. Delamare, O. Cugat, J. Meunier-Carus (2004). Magnetic bistable micro-actuator with integrated permanent magnet. Proceedings of IEEE Sensors 2004, Vienne

Faure, F., J. Delamare (1999). Low cost magnetic bearings. Proceedings of ISEM 1999

Guiziou, R., (2002). http://artemmis.univmrs.fr/cybermeca/Formcont/mecaspa/SCAO/QU ATERN/QUATERNO.HTM

Rostaing H., J. Stepanek, B. Delinchant, J. Delamare, O. Cugat (2004). Magnetic, out-of-plane bistable $\mu$-actuator. Vol. no. 2, in: Proceedings of the international workshop on High Performance Magnets and their Applications, pp. 929-935

Stepanek, J., H. Rostaing, J. Delamare, O. Cugat (2003). Fast dynamic modeling of magnetic micro-actuator. Vol. no. 272-276P1, in: Journal of Magnetism and Magnetic Materials, Proceedings of the International Conference on Magnetism pp 669-671

Stepanek, J., H. Rostaing, B. Delinchant, J. Delamare, O. Cugat, (2004). 3D calculation of forces and torques affecting permanent magnets - application to microsystems, submitted to Sensors \& Actuators, Physical A 